\title{
Chitosan Derivatives as Biosorbents for Basic Dyes
}

\author{
Nikolaos K. Lazaridis*, George Z. Kyzas, Alexandros A. Vassiliou, Dimitrios N. Bikiaris
}

Division of Chemical Technology, School of Chemistry Aristotle University, GR-541 24, Thessaloniki, Greece

\section{Supporting Information Available \\ Experimental Preparation}

For the preparation of the grafted chitosan with poly (acrylamide) (Ch-g-Aam) an exact amount of chitosan (Ch, 2.25 $\times 10^{-6}$ moles) was initially dissolved in a $2 \mathrm{v} / \mathrm{v} \%$ acetic acid aqueous solution followed by the addition of a solution of the monomer (Aam, 3.38x $10^{-2}$ moles). This was followed by the addition of a solution of the initiator (KPS, $5 \times 10^{-4}$ moles). The final solution $(50 \mathrm{~mL})$ was poured into a $100 \mathrm{~mL}$ stoppered flask, which was then placed in a thermostated bath at the desired reaction temperature (333 K) for 45 min. Prior and during the polymerization reaction the solution was purged with argon. After the completion of the grafting reaction, the polymerization mixture was rapidly cooled down to ambient temperature and neutralized to $\mathrm{pH} 8$ with the addition of a $1 \mathrm{~N} \mathrm{NaOH}$ solution. While stirring, the gelled was poured into a large amount of acetone. After $24 \mathrm{~h}$, satisfactory dewatering was achieved and the hardened gel particles were filtered and exhaustively extracted with a methanol: water (7:3) solution in order to remove the unreacted monomer, initiator and its by-products and the poly (acrylamide) (PAam) homopolymer that could eventually be formed during the grafting reaction. The dried remaining product at $323 \mathrm{~K}$ was considered the grafted copolymer. 
Chitosan grafted with poly (acrylic acid) (Ch-g-Aa) was prepared in a similar manner. Chitosan $\left(8.45 \times 10^{-7}\right.$ moles) was dissolved in an aqueous solution of the monomer ( $A a, 4.86 \times 10^{-2}$ moles) after which the addition of a solution of the initiator (KPS, $2 \times 10^{-4}$ moles) followed. A $100 \mathrm{~mL}$ stoppered flash containing the above solution (20 mL) was placed in a thermostated bath (343 K) for $2 \mathrm{~h}$ during which the reaction was carried out in the homogenous phase. Prior and during the polymerization reaction the solution was purged with argon. After rapid cooling down to room temperature, neutralization to $\mathrm{pH} 8$ and dewatering in acetone (24 h), a purification stage ensued by extraction with methanol in a Soxhlet apparatus for $24 \mathrm{~h}$ leading to the removal of any unreacted monomer, initiator and its by-products and the eventually formed poly(acrylic acid) (PAa) homopolymer. After drying at $323 \mathrm{~K}$ the grafted copolymer was obtained.

The biosorbent particles were chemically cross-linked with the bifunctional reagent glutaraldehyde (GA). The granulated products were suspended in an aqueous glutaraldehyde $5 \times 10^{-3} \mathrm{~mol} / \mathrm{L}$ solution with the use of a magnetic stirrer at a concentration of 1 wt $\%$, which corresponded to approximately 2:1 GA aldehyde groups (-CHO) per initial chitosan amine groups $\left(-\mathrm{NH}_{2}\right)$. The reactions were carried out at ambient temperature for a period of $24 \mathrm{~h}$. Extensive rinsing of the granulated chitosan particles with hot deionized water ensured the removal of any unreacted GA. Finally, the particles of $(C h) c,(C h-g-A a) c$ and $(C h-g-A a) c$ were dried at $323 \mathrm{~K}$. 